\title{
Summary PART I
}

In Part I, I introduced a set of material-discursive associations that make casework possible. First, I described associations for 'knowing' what asylum casework is about, namely through policy, legal, and organisational framings; and legal devices, classifications, and ways of 'knowing asylum' through heuristics and exemplars. Second, I presented associations for 'doing' asylum casework and thus "act[ing] in the name of the state" (Gupta 1995) namely positionality and membership devices, techniques of super-vision and re-collecting collectives; and key devices that mediate particular facets of the work of assembling cases like recording, inscription, coordination, and writing devices.

In terms of the asylum dispositif, I argued that it becomes stabilised - and is in a way constituted - in material-discursive associations such as those introduced in Part I. First, I provided insights into some crucial framings of the asylum dispositif which allow caseworkers to situate their practice - and ultimately to make sense of the various rationalities that inform them (see subchapter 8.2). I suggested some of the ways in which the governing of asylum is crucially entangled in the "relational politics of (im)mobilities" (Adey 2006) underlying much of Swiss (and European) migration policy. I turned to the evolution of asylum law and highlighted some important 'constants' of much legislative activism of the last decades, such as the acceleration of procedures and the deterrence rather than legal protection of asylum seekers. I further situated practices of case-making in an asylum "office on the move" (Fieldnotes): an administration that went through various reorganisations in the last years, and at the brink of a substantial restructuration of the Swiss procedure.

Considering knowledge practices involved in case-making, I suggested that they all converge in the need to resolve asylum cases in administrative 
orders called decisions". I provided a glimpse in the basic notions of arriving at this decision": the facts of the case" and the considerations". The production of the facts" involves the evaluation of evidence and conducting of hearings with applicants. The writing of the considerations* involves argumentation in terms of (at least) two core provisions of Swiss asylum law: the refugee definition (Article 3) and credibility (Article 7). I have suggested that how to resolve cases in these terms requires essentially practical knowledge which can be usefully thought of in terms of heuristics and exemplars. Heuristics, as embodied rules of thumb (Gigerenzer 2013), offer crucial simplifications of the complex legal and organisational conceptual landscape. While heuristics 'boil down' law and policy to its pragmatic 'essences', it is only through caseworkers' encounters with concrete cases that they start 'making sense' of the terms of governing asylum. Such concrete cases operate as exemplars in a Kuhnian (1967) sense, as they exemplify the meaning of abstract theories and concepts. In the interplay of heuristics and exemplars, caseworkers develop a sense of how to best navigate new cases they are supposed to (partially) assemble or resolve.

I have furthermore cautioned against taking agency for case-making for granted, but instead considering the intricate and indeterminate "agentic formations" in which the asylum dispositif becomes (re)assembled. Such agentic formations entail that caseworkers - who are both indivisibly human and well-equipped and assembled in larger collectives (at least the nation and the office) - become enabled for case-making. Equipment, on the one hand, serves their incorporation in collectives of case-making; on the other hand, it provides them with the material-discursive 'means' for case-making. I have introduced crucial equipment for incorporation, such as membership devices (including keys, badges and smartcards) that allow caseworkers' bodily and virtual access to and circulation in the space-times of case-making. Super-vision and meetings can be considered technologies for assembling and enacting particular collectives of case-making (superior-caseworker super-vision for case resolutions; or meetings for, for instance, enrolment in calculative collectives).

In the last subchapter, I introduced some crucial technologies for making cases in such assemblies. I suggested distinguishing between technologies and material-discursive devices in terms of their capacities of recording, inscription, coordination, and writing. For instance, I suggested that the technology of pagination and devices of file registers enable the inclusion 
and exclusion of documents as records in case files, indicate the procedural stage of a case, and limit the release of records (by, for instance, classifying some as internal or confidential). I considered hearings as technologies of inscription by enabling the selective import of 'realities of the applicant's lives' into the case. In this view, protocols are crucial inscription devices for turning situated dialogical events into textual records detached from the social context of their constitution. An important technology of coordination that I introduced is the asylum practice*. Asylum practice* guidelines, called APPA, crucially mediate how applicants are encountered by formatting 'realities of flight', for instance, in terms of (ir)relevant persecution scenarios. When it comes to technologies of writing, I introduced crucial writing devices for assembling decisions*: standard letters and boilerplates. These not only make writing practices more efficient, as I suggest, but become crucial mediators for the legal and argumentative pathways caseworkers consider to exist.

In Part II, I turn to the pragmatics of governing asylum: the situated practices and considerations of case-making. 
